\title{
Low-loss subwavelength plastic fiber for terahertz waveguiding
}

\author{
Li-Jin Chen, Hung-Wen Chen, Tzeng-Fu Kao, Ja-Yu Lu, and Chi-Kuang Sun \\ Department of Electrical Engineering and Graduate Institute of Electro-Optical Engineering, \\ National Taiwan University, Taipei 10617, Taiwan
}

\begin{abstract}
Received September 2, 2005; revised October 14, 2005; accepted October 18, 2005
We report a simple subwavelength-diameter plastic wire, similar to an optical fiber, for guiding a terahertz wave with a low attenuation constant. With a large wavelength-to-fiber-core ratio, the fractional power delivered inside the lossy core is reduced, thus lowering the effective fiber attenuation constant. In our experiment we adopt a polyethylene fiber with a $200 \mu \mathrm{m}$ diameter for guiding terahertz waves in the frequency range near $0.3 \mathrm{THz}$ in which the attenuation constant is reduced to of the order of or less than $0.01 \mathrm{~cm}^{-1}$. Direct free-space coupling efficiency as high as $20 \%$ can be achieved by use of an off-axis parabolic mirror. Furthermore, all the plastic wires are readily available, with no need for complex or expensive fabrication. (C) 2006 Optical Society of America

OCIS codes: $060.2430,260.3090$.
\end{abstract}

Strong attenuation in the terahertz $(\mathrm{THz})$ gap, which occupies the wavelength range from 30 to $3000 \mu \mathrm{m}$ (10 to $0.1 \mathrm{THz}$ ), is always a severe problem in longdistance $\mathrm{THz}$ waveguiding. Even though much research on $\mathrm{THz}$ generation and detection techniques has been done to improve the detectable level of the $\mathrm{THz}$ signals, the unacceptably high dielectric loss and finite metal conductivity in this regime are still challenging for optical fiber-type or metal-type waveguide design. Hence almost all the setups for current $\mathrm{THz}$ application systems are constructed with planar or curved metal reflectors, which make the systems not only rigid but also vulnerable to environmental disturbance. With the need for a compact, reliable, and flexible $\mathrm{THz}$ system for various applications, ${ }^{1,2}$ a low-loss $\mathrm{THz}$ waveguide is essential. Recently, many metal-based waveguiding techniques, including the use of metal parallel plates ${ }^{3}$ and bare metal wires, ${ }^{4,5}$ were reported to have much improved attenuation constants compared with other, conventional microwave waveguides ${ }^{6,7}$ and various dielectric-based waveguides. ${ }^{8-11}$ Even though current reports show that the metal-based guiding technique is the best solution to meet the need for low attenuation, we find that a simple plastic wire with a subwavelength diameter, similar to an optical fiber, can also provide a low guiding attenuation in single-mode operation. The concept of such low-loss waveguiding was previously demonstrated at both microwave ${ }^{12-14}$ and optical $^{15}$ frequencies. In this Letter, we demonstrate a low-loss plastic fiber for $\mathrm{THz}$ waveguiding. In our study we adopted a $200 \mu \mathrm{m}$ diameter polyethylene (PE) wire as the subwavelength $\mathrm{THz}$ fiber for a frequency near $300 \mathrm{GHz}$. With a small diameter-towavelength ratio, a large portion of $\mathrm{THz}$ waves is guided outside the lossy core such that the attenuation constant is successfully reduced to be of the order of or less than $0.01 \mathrm{~cm}^{-1}$, while the measured free-space coupling coefficient of the fiber can reach up to the order of $20 \%$ when an off-axis parabolic mirror is used. With direct coupling capability, our proposed $\mathrm{THz}$ fibers can easily be integrated with cur- rent $\mathrm{THz}$ systems, which can improve the systems' flexibility and reliability.

To simplify the analysis of the guiding characteristics of a subwavelength $\mathrm{THz}$ fiber we assume that the plastic wire, which has the same geometry as an aircladding fiber, has a circular cross section, a uniform radius $a$, and a step-index profile with inner refractive index $n_{1}$ and outer refractive index $n_{2}$. Since air serves as the cladding material, we assume that $n_{2}$ $=1$. By solving Maxwell's equation with the above parameters, one can analytically express the mode fields in the form of Bessel functions. ${ }^{16}$ However, because of the lack of a nonabsorptive material in the $\mathrm{THz}$ regime, all the modes confined within the dielectric core will be subject to material absorption, and the fiber will be too lossy to compete with a metalbased fiber. A straightforward way to improve the attenuation is to reduce the fractional power inside the dielectric core by using a subwavelength-diameter plastic wire with a small diameter-to-wavelength ratio, which will also ensure single-mode $\left(\mathrm{HE}_{11}\right)$ operation. ${ }^{16}$ To calculate the fractional power flow inside the core area, one has to solve Poynting vector $S_{z}$ in the direction of propagation ( $z$ direction). ${ }^{16}$ Figure 1 shows the calculated spatial distributions of the normalized $S_{z}$ of a $200 \mu \mathrm{m}$ diameter PE wire for 300 , 500,700 , and $900 \mathrm{GHz}$ with $n_{1}=1.5{ }^{17}$ It can be observed that the fractional power flowing outside the $\mathrm{PE}$ core decreases as the frequency of the guided waves increases. One can obtain fractional power $\eta$ inside the core by

$$
\eta=\frac{\int_{0}^{a} S z(r) r \mathrm{~d} r \mathrm{~d} \phi}{\int_{0}^{\infty} S z(r) r \mathrm{~d} r \mathrm{~d} \phi} .
$$

The value of $\eta$ can be used to estimate roughly the effective fiber attenuation constant influenced by the core absorption. For a small $\eta$, which happens when a small-diameter plastic wire is adopted, the attenuation constant will approach that of the air cladding, 


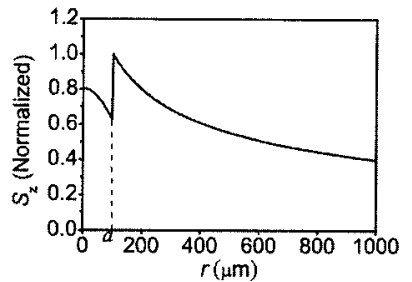

(a)

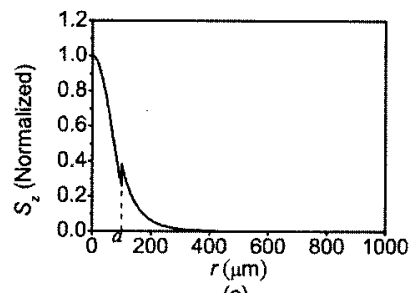

(c)

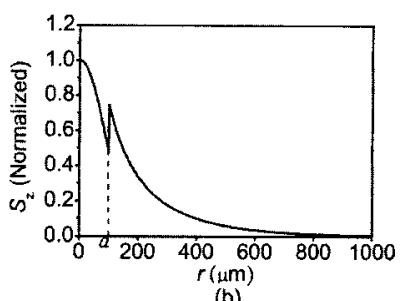

(b)

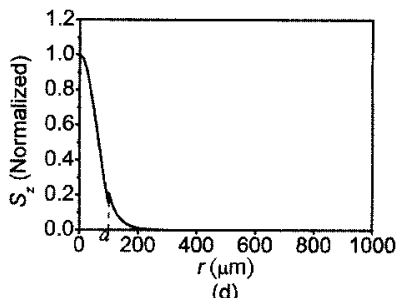

(d)
Fig. 1. Spatial distribution of the $z$-direction Poynting vector about a $200 \mu \mathrm{m}$ diameter $(a=100 \mu \mathrm{m})$ PE wire at frequencies of (a) 300, (b) 500, (c) 700, and (d) $900 \mathrm{GHz}$. The $\mathrm{PE}$ wire is assumed to be surrounded with air.

while for an $\eta$ close to 1 the attenuation constant will approximate that of bulk PE. To precisely estimate the fiber attenuation caused by the material absorption, we adopted a perturbation method. ${ }^{12}$ It assumes that the power loss per wavelength in the propagation is small compared with the total power flowing along the fiber, which is reasonable for a subwavelength plastic wire in the $\mathrm{THz}$ regime. Using the method simplified with the Poynting theorem, ${ }^{12}$ we can find fiber attenuation constant $\alpha_{f}$, given by

$$
\alpha_{f}=\left|\frac{1 \mathrm{~d} P}{P} \frac{\sigma \int|E|^{2} \mathrm{~d} \tau}{\mathrm{d} z}\right|=\frac{\sigma S_{z} \mathrm{~d} \tau \mid}{\mid \int}
$$

where $\tau$ is an infinite plane perpendicular to the fiber axis and $\sigma$ is the conductivity, which is related to the material absorption constant. For the plastic core, conductivity is a function of refractive index $n_{1}$ and the absorption constant of the plastic, $\alpha_{m}$, which can be expressed as $\sigma=n_{1} c \alpha_{m} / 4 \pi$. For air cladding, the conductivity approximates zero because of its negligible absorption of air. The calculated $\alpha_{f}$ as a function of electromagnetic frequency is shown as a dashed curve in Fig. 2(a), for which it is assumed that $\alpha_{m}$ $=1 \mathrm{~cm}^{-1}$.

We used $\mathrm{PE}$ wires for the subwavelength $\mathrm{THz}$ fibers because PE has a relatively low $\alpha_{m}$, with no significant absorption lines in the $\mathrm{THz}$ window. The experimental arrangement employed for the fiberattenuation measurement is shown in Fig. 3. The $\mathrm{THz}$ wave radiated from a photonic transmitter ${ }^{18} \mathrm{ex}-$ cited by an optical coherent control system ${ }^{19}$ to control the central frequency of radiation. The radiation was collimated and coupled into one end of the fiber by a pair of identical parabolic mirrors $(3.82 \mathrm{~cm}$ focal length). The fiber was held by a fiber coupler made from paper. The reason for choosing paper as a supporting material is its low absorption in the $\mathrm{THz}$ regime. The measured transmission power of the adopted individual paper in the $\mathrm{THz}$ wavelength of interest is $\sim 90 \%$. The coupler was controlled by a kinetic mount and a three-dimensional (3D) stage for position and tilt adjustments. The $\mathrm{THz}$ power delivered by the fiber was collected by a metal cone and detected by a translation-stage-mounted Si bolometer. The metal cone was used to resize the detection window to a small aperture to prevent scattered waves from being detected. We attached a paper fiber output holder to the input of the cone. A hole that pierced the center of the paper was slightly smaller than the cross section of the fiber core to ensure tight fiber mounting.

Since our collected PE wires came from the fishing industry and had a relatively poor material quality and many bends that seriously degrade their guiding performance, the fiber to be measured was first heated and straightened with steam, which made $\alpha_{m}$ higher than expected. The ends of the fiber were polished with sandpaper to enhance coupling efficiency. For consistency, the measurement of the attenuation constant was performed with the same PE fiber. Three fiber lengths, $17.5,13.5$, and $6 \mathrm{~cm}$, were measured. We began with the measurement of output $\mathrm{THz}$ power for the longest length by using a bolometer and a lock-in amplifier at different radiation frequencies. Then we cut a small piece of fiber off the output end and repeated the measurements. To minimize measurement errors and to ensure the same extent of paper contact during the measurement, we did not remove the fiber from its input coupler. We made a new hole in the output paper holder for every measured length to keep the fiber output end tightly

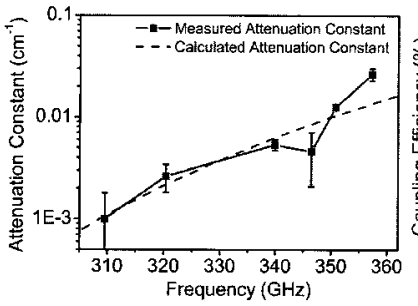

(a)

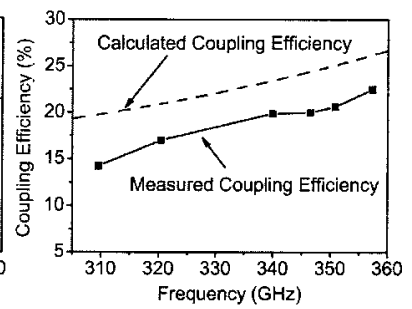

(b)
Fig. 2. (a) Measured fiber attenuation constant of the $200 \mu \mathrm{m}$ diameter $\mathrm{PE}$ wire in the frequency range 310-360 GHz. For comparison, the calculated fiber attenuation constant of an ideal $\mathrm{THz}$ fiber, whose absorption constant $\alpha$ is assumed to be $1 \mathrm{~cm}^{-1}$ in this frequency range, is shown. (b) Comparison of measured and calculated coupling efficiency of the $\mathrm{PE}$ wire in the frequency range 310-360 GHz.

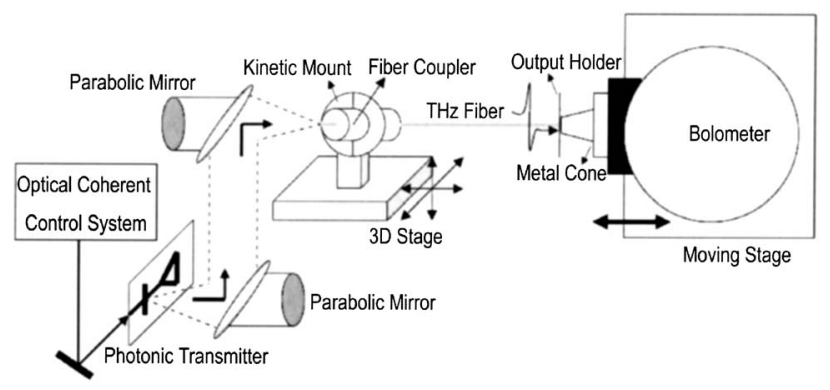

Fig. 3. Experimental setup of the $\mathrm{THz}$ fiber measurement system and the structure of a paper fiber coupler. 
mounted. For a sufficiently small core-diameter-towavelength ratio $\left(2 a / \lambda_{0}\right)$, the frequency of interest is $\sim 300 \mathrm{GHz}$. By analyzing the transmitted $\mathrm{THz}$ power for different fiber lengths we obtained the fiber attenuation constant. In Fig. 2(a) we show the experimentally obtained attenuation constant as a function of frequency from 310 and $360 \mathrm{GHz}$, corresponding to the $2 a / \lambda_{0}$ ratio from 0.20 to 0.17 . Similarly to the theoretical expectation, with a higher $\mathrm{THz}$ frequency and a larger $\eta$ the measured $\alpha_{f}$ increases with frequency for a fixed-diameter $\mathrm{PE}$ wire. Tuning the $\mathrm{THz}$ frequency from 310 to $360 \mathrm{GHz}$ results in a tenfold increase in the measured $\alpha_{f}$ value, from the order of $10^{-3}$ to the order of $10^{-2} \mathrm{~cm}^{-1}$, as expected from the theoretical calculation. It is important to note that this is to our knowledge the first time that a $\alpha_{f}$ value of the order of or less than $10^{-2} \mathrm{~cm}^{-1}$ was achieved for a $\mathrm{THz}$ fiber.

In fiber optics, coupling efficiency is always an important issue. This efficiency describes the power transfer from free space to the fiber that can be evaluated by an overlapping integral of the incident field and the mode field, which is operationally defined as follows:

$$
\eta_{\text {coupling }}(\omega) \frac{\left|\int E_{\text {input }}(\omega) E_{\text {mod } e}{ }^{*}(\omega)^{2} \mathrm{~d} S\right|^{2}}{\int\left|E_{\bmod e}(\omega)\right|^{2} \mathrm{~d} S \int\left|E_{\text {input }}(\omega)\right|^{2} \mathrm{~d} S},
$$

where $E_{\bmod e}(\omega)$ is the fiber mode field of a specific frequency and $E_{\text {input }}(\omega)$ is the field distribution of incident waves. To numerically estimate coupling efficiency with a 2 -in. parabolic mirror, we assume that the input wave has a Gaussian distribution with a focal diameter of $4 \lambda F / \pi$, where $\lambda$ is the wavelength and $F$ is the nominal focal ratio (or $F$-number) that is the ratio of the mirror's focal length $f$ to its aperture $d .^{20}$ In our case, the focal length is $3.82 \mathrm{~cm}$, which makes the diameter of the focal point $\sim 0.96 \lambda$. With these parameters, the coupling efficiency of the $\mathrm{PE}$ wire under ideal conditions in the frequency range $310-360 \mathrm{GHz}$ can be calculated. In experiments, we determined the coupling efficiency by measuring the ratio of the output-to-input power and then calibrating it with the loss caused by the paper coupler, the output paper holder, and the attenuation of the fiber. Both the theoretical and the measured values are shown in Fig. 2(b). As was theoretically predicted, with increased $\mathrm{THz}$ frequency the coupling efficiency is improved under our experimental arrangement because of improved mode-field overlap. A direct coupling efficiency as high as $20 \%$ was experimentally achieved. However, the relatively constant difference near $5 \%$ between two curves implies some systematic errors during the experimental estimation, such as neglect of the collective loss of the metal cone and of the input-output coupling reflections. These effects cause an underestimation of the measured coupling efficiency.

In conclusion, we have demonstrated a low-loss $\mathrm{THz}$ subwavelength fiber with $\mathrm{PE}$ wires that are readily and inexpensively available. With a direct coupling capability, our proposed $\mathrm{THz}$ fiber has the potential to be integrated with current $\mathrm{THz}$ systems and could also be applied to evanescent wave fiber sensing. Because of the weakly guiding nature of the fiber, the guiding attenuation is successfully reduced.

This work was sponsored by the National Science Council of Taiwan under grant 93-2215-E-002-040 and by the National Taiwan University Center for Genomic Medicine. C.-K. Sun's e-mail address is sun@cc.ee.ntu.edu.tw.

\section{References}

1. D. M. Mittleman, R. H. Jacobsen, R. Neelamani, R. G. Baraniuk, and M. C. Nuss, Appl. Phys. B 67, 379 (1998).

2. D. M. Mittleman, R. H. Jacobsen, and M. C. Nuss, IEEE J. Sel. Top. Quantum Electron. 2, 679 (1996).

3. R. Mendis and D. Grischkowsky, Opt. Lett. 26, 846 (2001).

4. K. Wang and D. M. Mittleman, Nature 432, 376 (2004).

5. T.-I. Jeon, J. Zhang, and D. Grischkowsky, Appl. Phys. Lett. 86, 161904 (2005).

6. M. Y. Frankel, S. Gupta, J. A. Valdmanis, and G. A. Mourou, IEEE Trans. Microwave Theory Tech. 39, 910 (1991).

7. J.-M. Heiliger, M. Nagel, H. G. Roskos, H. Kurz, F. Schnider, W. Heinrich, R. Hey, and K. Ploog, Appl. Phys. Lett. 70, 2233 (1997).

8. R. Mendis and D. Grischkowsky, J. Appl. Phys. 88, 4449 (2000).

9. S. P. Jamison, R. W. McGowan, and D. Grischkowsky, Appl. Phys. Lett. 76, 1987 (2000).

10. H. Han, H. Park, M. Cho, and J. Kim, Appl. Phys. Lett. 80, 2634 (2002).

11. M. Goto, A. Quema, H. Takahashi, S. Ono, and N. Sarukura, Jpn. J. Appl. Phys., Part 2 43, L317 (2004).

12. C. H. Chandler, J. Appl. Phys. 20, 1188 (1949).

13. W. M. Elsasser, J. Appl. Phys. 20, 1193 (1949).

14. C. Yeh, F. I. Shimabukuro, and J. Chu, Appl. Phys. Lett. 54, 1183 (1989).

15. L. Tong, R. R. Gattass, J. B. Ashcom, S. He, J. Lou, M. Shen, I. Maxwell, and E. Mazur, Nature 426, 816 (2003).

16. A. Yariv, Optical Electronics in Modern Communications, 5th ed. (Oxford U. Press, 1997).

17. J. R. Birch, G. J. Simonis, M. N. Afsar, R. N. Clarke, J. M. Dutta, H. M. Frost, X. Gerbaus, A. Hadni, W. F. Hall, R. Heidinger, W. W. Ho, C. R. Jones, F. Koniger, R. L. Moore, H. Matsuo, T. Nakano, W. Richter, K. Sakai, M. R. Stead, U. Stumper, R. S. Vigil, and T. B. Wells, IEEE Trans. Microwave Theory Tech. 42, 956 (1994).

18. M.-C. Tien, H.-H. Chang, J.-Y. Lu, L.-J. Chen, S.-Y. Chen, R.-B. Wu, W.-S. Liu, J.-I. Chyi, and C.-K. Sun, IEEE Photon. Technol. Lett. 16, 873 (2004).

19. A. S. Weling and D. H. Auston, J. Opt. Soc. Am. B 13, 2783 (1996).

20. M. Born and E. Wolf, Principles of Optics, 7th ed. (Cambridge U. Press, 1999), p. 200. 